\title{
BMJ Open A qualitative study exploring the factors influencing admission to hospital from the emergency department
}

\author{
Ian Pope, ${ }^{1}$ Helen Burn, ${ }^{2}$ Sharif A Ismail, ${ }^{3}$ Tim Harris, ${ }^{4}$ David McCoy ${ }^{5}$
}

To cite: Pope I, Burn H, Ismail SA, et al. A qualitative study exploring the factors influencing admission to hospital from the emergency department. BMJ Open 2017;7:e011543. doi:10.1136/ bmjopen-2016-011543

- Prepublication history and additional material for this paper are available online. To view these files please visit the journal online (http://dx.doi. org/10.1136/bmjopen-2016011543).

Received 16 February 2016 Revised 6 July 2016 Accepted 29 July 2016

\section{SLinked}

http://dx.doi.org/10.1136/ bmjopen-2016-011547

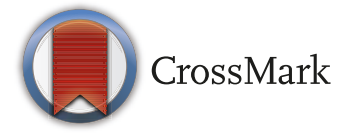

${ }^{1}$ Emergency Department, Royal London Hospital, London, UK ${ }^{2}$ Brighton and Sussex University Hospitals NHS Trust, Brighton, UK

${ }^{3}$ Barts Health NHS Trust and Queen Mary University of London, London, UK ${ }^{4}$ Emergency Department, Royal London Hospital, London, UK

${ }^{5}$ Centre for Primary Care and Public Health, Queen Mary University of London, London, UK

Correspondence to

Dr. lan Pope;

ianpope@gmail.com

\begin{abstract}
Objective The number of emergency admissions to hospital in England and Wales has risen sharply in recent years and is a matter of concern to clinicians, policy makers and patients alike. However, the factors that influence this decision are poorly understood. We aimed to ascertain how non-clinical factors can affect hospital admission rates.
\end{abstract}

Method We conducted semistructured interviews with 21 participants from three acute hospital trusts. Participants included 11 emergency department (ED) doctors, 3 ED nurses, 3 managers and 4 inpatient doctors. A range of seniority was represented among these roles. Interview questions were developed from key themes identified in a theoretical framework developed by the authors to explain admission decision-making. Interviews were recorded, transcribed and analysed by two independent researchers using framework analysis.

Findings Departmental factors such as busyness, time of day and levels of senior support were identified as non-clinical influences on a decision to admit rather than discharge patients. The 4-hour waiting time target, while overall seen as positive, was described as influencing decisions around patient admission, independent of clinical need. Factors external to the hospital such as a patient's social support and community follow-up were universally considered powerful influences on admission. Lastly, the culture within the ED was described as having a strong influence (either negatively or positively) on the decision to admit patients.

Conclusion Multiple factors were identified which go some way to explaining marked variation in admission rates observed between different EDs. Many of these factors require further inquiry through quantitative research in order to understand their influence further.

\section{INTRODUCTION}

During the past decade, acute hospital admissions in the National Health Service have been increasing, growing by $37 \%$ between 2002 and 2012 in England. ${ }^{1}$ A variety of factors have contributed to this increase, including a growing and ageing population, with a consequent rise in disease prevalence. ${ }^{2}$ However this does not explain all of the increase. If admission numbers continue to grow at current rates, it could lead to an

\section{Strengths and limitations of this study}

This study is the first to qualitatively explore the organisational and policy factors that influence clinical and management practice within emergency departments (EDs) and their possible links to avoidable hospital admissions.

- Semistructured interviews of 21 informants from three EDs, including consultants, junior doctors, nurses and managers, allowed for data to be collected from multiple viewpoints.

- An iterative process of data collection and analysis was used to produce a framework of the possible determinants of clinical and management practice that can be used to describe and analyse clinical and management practice in other hospital EDs.

- This study was conducted in parallel with a quantitative study examining the risk factors for admission, which allowed cross-checking of results.

- The main limitation of this study is that its findings are of limited external validity.

additional 6.2 million hospital bed days per annum by 2022. ${ }^{2}$ Clinicians, managers and policy makers are now under pressure to slow down the overall increase in hospital admissions, particularly in terms of acute admissions that are deemed avoidable or unnecessary.

There is considerable variability in the proportion of emergency hospital attendances which result in admissions, ranging from $12 \%$ to $48 \%$ with a median of $24 \%$, in England. ${ }^{3}$ A large amount of this variation is explained by factors beyond the control of health services such as employment rates, deprivation levels and age profile. However, some of the variation is due to health system factors that affect the admissions process. One recent analysis of admissions in England found that $28 \%$ of the variation in rates of avoidable hospital admissions was due to health system factors. ${ }^{4}$

Another study of the variation in the rate of avoidable emergency admissions in England found poor availability of general practice out-of-hours services, lack of availability of 
senior review within emergency departments (EDs), variation in how patient admissions were coded in view of the 4-hour target, and a lack of services to facilitate discharge from the ED accounting for some of the variation in avoidable admissions. ${ }^{5}$ Similarly, a study of an ED in Ottawa, Canada identified triage; diagnosis and communication; the place of patient assessment in an ED; and crowding as factors that influenced whether a patient is admitted or discharged. ${ }^{6}$

Research aimed at reducing hospital admission rates has largely focused on interventions aimed at primary care; for example, improving continuity of care with General Practice (GP), introducing telemonitoring, dedicated specialist nursing initiatives, hospital at home and enhancing self-management. ${ }^{7}$ However, a neglected aspect of a patient's journey is the period that a patient spends in the ED prior to admission. Only a few studies have examined the potential to reduce the conversion rate of $\mathrm{ED}$ attendance to admission, and have mainly focused on access to senior review and multidisciplinary team input in the ED. ${ }^{5}$

This paper presents the findings from a qualitative study of three EDs aimed at identifying and understanding those factors related to the management and workings of an ED that may influence the incidence of avoidable or unnecessary hospital admissions, and which may be amenable to policy or managerial intervention.

\section{METHODS}

\section{Study sites}

Three hospital EDs belonging to a single NHS Trust in London formed the basis for this study. Site 1 was an ED and Major Trauma Centre in central London with a specialist stroke unit. Sites 2 and 3 were EDs in District
General London hospitals. ST segment elevation myocardial infarctions are treated at a separate cardiology centre that was not included in this study. The population served by the three EDs is diverse but on the whole is relatively underserved and ethnically mixed when compared with the English population.

\section{Data collection and analysis}

To help collect data, a framework of factors that may influence the decision to admit a patient from an ED was developed (see figure 1). This was based on the working knowledge of DM and IP, as well as a review of the limited literature on the effect of ED-related factors on hospital admission rates. ${ }^{56}$ The framework postulated a model that placed the individual doctor as the final decision-maker, but influenced by four sets of other factors: those related to the patient; the ED; the hospital; and the wider policy and health system environment. This framework formed the basis for the design of a semistructured key informant interview schedule that was used to collect data.

Individual semistructured interviews, apart from one group interview with three informants (at their request), was the primary method used to collect data about the factors influencing the decision to admit patients as this allowed a variety of professionals with considerable time constraints to fully present their views and experiences. Ideally, our one-to-one interviews would have been complemented with focus group discussions (to enable a discussion about the factors identified) and a survey (potentially to enable the involvement of a larger group of informants). However, this was not feasible due to resource constraints.

The semistructured interview guide was piloted on one informant and adjusted in minor ways in light of the experience. The interviewer stressed the fact that the focus of

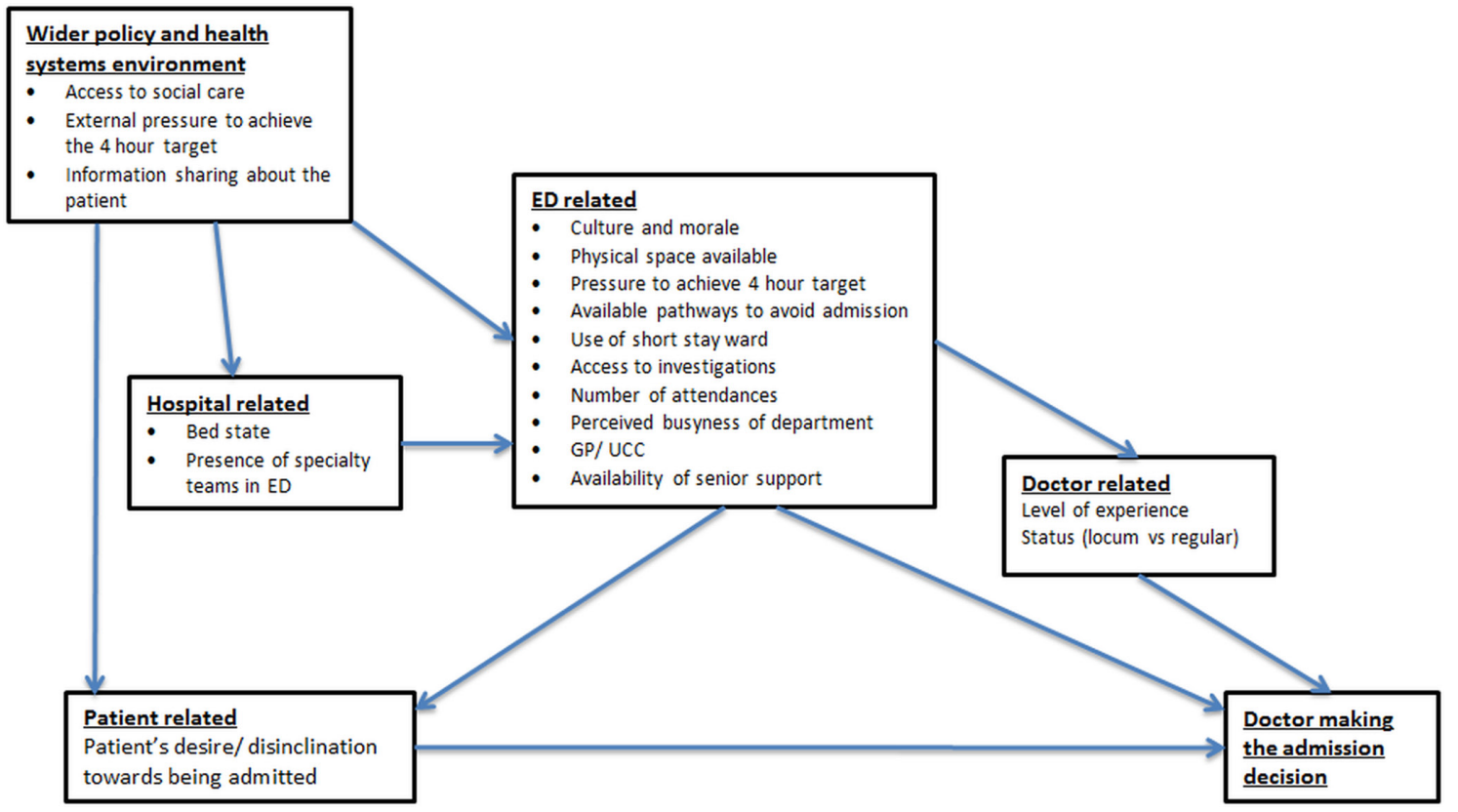

Figure 1 Framework used to shape semistructured interview schedule. ED, emergency department. GP, General practitioner. UCC, Urgent Care Centre. 
Table 1 Study informants and site

\begin{tabular}{llll}
\hline & \multicolumn{3}{l}{ Emergency medicine } \\
\cline { 2 - 4 } & Site 1 & Site 2 & Site 3 \\
\hline Consultant & 2 & 3 & 1 \\
Registrar & 2 & & \\
Senior house officer & 1 & 1 & 1 \\
Nurse/manager & 1 & & \\
Nurse & 2 & 1 & \\
Manager & \multicolumn{4}{l}{1} \\
\hline \multirow{2}{*}{ Acute medicine } & \\
\cline { 2 - 4 } Consultant & Site 1 & Site 2 & Site 3 \\
\hline
\end{tabular}

the research was on non-clinical reasons for admission, though informants were free to raise any clinical issues if they sought to.

Altogether, 31 potential informants, presenting a mix of clinicians and managers, were purposively invited to participate from all three EDs. They were contacted by email and provided with an information sheet about the study. A total of 15 doctors, 3 nurses and 3 managers ended up being interviewed. Of the 15 doctors, 10 were consultants ( 6 of whom were emergency medicine consultants and four of whom worked on acute inpatient medical wards); 2 were specialist registrars; and 3 were senior house officers. Due to a variable response rate, a limitation of this study is that an equal spread of informants across the three EDs was not achieved (see Table 1). However, some of the informants had previously worked across multiple sites so were able to provide insight into how different sites functioned.

IP conducted all the interviews. At the time of the study IP was working as an Academic Foundation Programme doctor but had previously worked as a junior doctor in medicine at site 2 and as a junior doctor in the EDs of sites 1 and 3. This provided the study with some 'insider knowledge' which enhanced the quality and validity of data collected and also enabled access to a more open discussion with the informants. However, IP's 'insider knowledge' and experience of having previously worked with eight of the informants in various capacities required a conscious effort to minimise any potential for bias or subjectivity being introduced in the interviews.
Box 1 Key factors determining the rate of avoidable or unnecessary admissions derived from analysis of interview transcripts

\section{Key factors}

Four-hour waiting time target

Availability of services to enable safe and effective care at home

Availability of diagnostic and outpatient alternatives

Clinical staffing and workload

Departmental culture

Response to patient expectations and preferences

Data were collected primarily in the form of free speech responses to open or semiopen questions. The interviews took place in the informant's places of work and lasted around $40 \mathrm{~min}$. One participant requested the opportunity to review the transcript of their interview, which was provided. The interviews were digitally recorded and transcribed.

The individual transcripts were then reviewed, manually coded and analysed jointly by IP and DM. The purpose of the analysis was to identify those factors related to the way the ED is managed and operated which were deemed to be important in determining the rate of avoidable or unnecessary admissions. Following this, it was felt that the same transcripts should be reviewed, coded and analysed independently by a third person (HB). The two sets of factors identified from the transcripts were then reviewed jointly and discussed. This led to the final development of a set of seven factors deemed to be important in determining the rate of avoidable or unnecessary admissions.

\section{Ethics}

Written consent was gained from all informants including consent to digitally record the interviews and to use position and site when using their quotes; this was granted by all informants. All data were anonymised and kept in a secure format.

\section{FINDINGS}

Interviews were carried out across three sites. A comparison of these sites is shown in Table 2.

Analysis of the data suggested a set of seven broad factors that were perceived by key informants to influence clinical decision-making in a way that might influence

Table 2 Comparison between the observation units at the three hospital sites

\begin{tabular}{llll}
\hline & Site 1 & Site 2 & Site 3 \\
\hline Ward & Clinical Decisions Unit & Clinical Decision Unit & Observation Unit \\
No. of beds & 14 & 12 & 25 \\
Staffing & ED consultant (daytime) & ED consultant (daytime) & Shared-care inpatient hospital \\
& ED registrar & ED registrar & specialties and ED \\
Conversion rate & $24 \%$ & $43.5 \%$ & $34 \%$ \\
\hline
\end{tabular}

ED, emergency department. 
the rate of avoidable or unnecessary admissions (box 1). Many, but not all, were preidentified by the framework used to shape the interview schedules. Each of these factors is discussed at greater length below.

\section{Four-hour waiting time target}

Most informants from all three sites agreed that the government directive introduced in 2000 that "no one should wait more than 4 hours in the ED from arrival to admission, transfer or discharge ${ }^{, 8}$ has had a major impact on EDs. ${ }^{9}$ The general view was that the 4-hour target has had positive effects including: increased resources for the ED, reduced waiting times, improved patient flow and increased awareness in the rest of the hospital about the challenges faced by the ED. According to one ED consultant, the challenge of meeting the 4-hour target had helped bring clinicians and managers together into 'a really tight group of seniors who are driving that department and really making something of that...".

However, informants described how the 4-hour target led to some patients being admitted before they had been fully assessed, as illustrated by the following quotes: 'If $a$ patient is going to breach because a blood result isn't back I'll refer to a specialty, even if they don't need to be admitted'; and 'sometimes you think you might be able to sort them out in $20 \mathrm{~min}$, but you only have 5 so they will have to be admitted' (acute medical consultant). A concept that came up several times was the '3:59 patient': a patient who is admitted because they are about to breach the target. Many of the ED doctors interviewed felt that a number of admissions could be avoided if they had had an extra hour or two to manage patients.

Another issue related to the 4-hour target which was incidental to the focus of the research on avoidable and unnecessary admissions but which was felt to be important was the effect that the 4-hour target had on impinging on the ability to comprehensively manage patients, with a greater emphasis being placed on clinical triage rather than on actual treatment. This also had an effect on the type of clinical exposure and practice that ED doctors experienced. In the words of one ED consultant: 'we do not get as much exposure to managing sick people now as we have to pass them on to other disciplines'; while an ED registrar stated that the target 'undermines the specialty...we are the best people to manage patients in the initial stages of their illness, but in reality we are referring patients elsewhere for this care because of the need to keep the flow going'.

\section{Availability of services to enable safe and effective care at home}

The ability of the ED to ensure adequate and safe social support and care at home was strongly identified as a factor perceived to affect admissions practice: 'sometimes you have to admit patients because you're unclear whether they'd be safe at home' (ED senior house officer). Another ED doctor stated that 'social admissions' are 'very common'. Three informants offered a percentage of admissions that they believed to be 'social' and gave figures ranging from $15 \%$ to $25 \%$.
Various informants stated that the ability to avoid so-called 'social admissions' was hindered by poor communication between the ED and community and social service providers, which prevented care packages and other forms of support being organised for patients who might otherwise have been discharged home. According to one medical consultant 'historical problems with social services support' mean that some patients are admitted for a short period of time as a fall-back. Another issue noted by some informants was a lack of availability of transport to take patients home could also result in some patients being admitted unnecessarily.

Concerns about a lack of care or support at home or an inability to arrange such care or support were said to be more pronounced over the weekend when, according to one ED senior house officer, 'you're much more likely to admit because they're going to be on their own, and you're not going to be able to get services started again until after the weekend'.

\section{Availability of diagnostic and outpatient alternatives}

Most informants identified a lack of access to rapid diagnostic services or to previous patient notes and clinical information, particularly when combined with the pressure of the 4-hour target, as being a cause for potentially avoidable or unnecessary admissions. According to a manager who had worked on all three sites, the unavailability of outpatient investigations was a particular problem on site 2 where 'there is a reliance on admitting to investigate'. An ED consultant also stated that 'greater availability of hot clinics' (ie, clinics where ED patients can be seen rapidly by a consultant rather than being admitted) would greatly help avoid admissions.

The time of attendance at an ED was described as a cofactor, with access to information or certain diagnostic procedures being more difficult after normal working hours. In the words of one of the ED consultants: ‘... during the day, you just scan; if normal, they go home; whereas out of hours, you keep them in to have a scan in the morning'. According to an ED senior house officer, 'at 9 am you have the ability to take a collateral history ... contact the GP, find out about services and get services set up', but this was said to be difficult to do out of hours.

Several informants also described how problems and difficulties in organising outpatient follow-up meant that clinicians were more likely to feel that it was better and safer to admit. One ED consultant stated that out of hours there is a lack of alternatives... when services are available, patients might be able to be referred to the ambulatory pathways... but out of hours these aren't available and patients are more likely to be admitted.' A medical consultant also noted that 'sometimes you think you might be able to sort something out as an outpatient, but then the structures we have in place in terms of follow up and getting results back are quite frail so sometimes it's just safer to admit a patient to do the tests', while another medical consultant stated that 'if there were better follow-up clinics, I maybe would have thought: 'no, you can come to clinic in 2 days' rather than be admitted'. Similarly, an ED registrar explained that long waits and bureaucratic difficulties in 
arranging further investigations through a patient's GP meant that it was sometimes in the patient's interest to be admitted.

\section{Clinical staffing and workload}

Clinical seniority and experience, including the availability of senior support for junior doctors, was a common theme in many interviews. It was described that clinicians with more experience are more likely to be risk-comfortable and send patients home, while junior doctors were more likely to admit because it was felt to be safer, especially when there is no senior support available. One ED senior house officer illustrated this point well: If $a$ consultant is the one who assesses them they would be much more confident in their clinical decision making... if I was unable to get a registrar or consultant to see (the patient), I would err on the side of caution... more likely to refer them (and admit)." According to an ED consultant 'the more senior the decision maker is the less likely you are to get admitted' due to 'a mixture of confidence and experience' while a medical consultant described how 'the longer you've been a doctor the more likely you are to take risks'.

It was also noted that there are generally fewer senior doctors available outside of normal working hours, and that junior doctors were likely to be more risk-averse towards the end of the working day. According to a medical consultant, 'I think psychologically for the doctor, if you're the last one to assess (the patient) and you say you can go home and it's 11 pm, you've got to be a very confident consultant to do that'. According to a junior ED doctor (senior house officer), 'if it's towards the end of the day or at night, you're more likely to admit someone than at 9am'. One medical consultant suggested that ' $70 \%$ (of patients) might, if there had been someone more senior available at the time, have been sent home (during the night)'.

The importance of clinical experience and confidence being important in potentially preventing avoidable or unnecessary admissions was also noted in relation to staffing levels and the number and clinical severity of patients. One of the ED consultants explained that high patient numbers lead to there being fewer staff available to carry out investigations and the actions needed to allow for a safe discharge: 'there's not enough doctors or nursing staff to perform all the things that are needed to decide whether someone can go home safely. Additionally, a high patient to staff ratio would often mean more junior doctors without senior support, leading to 'a lower threshold to admit' (ED registrar).

According to one ED nurse, 'when it's more chaotic, people make poorer decisions; when it's calm, people have more time and resources to make a decision'. Another informant stated that when it is busy, the quickest way of moving patients is to admit, rather than discharge: 'when it gets busy...try to clear space... to avoid the breach, the registrars might just do a really quick examination and refer to the medics.' (ED senior house officer). At the same time, it was noted that that when the wards are busy, the reverse can happen. In such instances, there is pressure to avoid admission and discharge patients, leading to a 'bigger problem that people are potentially inappropriately discharged' (ED consultant).

\section{Departmental culture}

Many informants felt that departmental culture could have a significant influence on admission practices, and potentially affect the rate of avoidable or unnecessary admissions. This was understood to include a combination of the attitudes of senior clinicians, managers and nurses; levels of motivation; and relationships between staff within the ED.

One ED consultant who had worked on all three sites stated that culture has a 'very large effect...I think the culture does influence why you admit people. He explained that, for example, 'a more horizontal hierarchy is much better in departmental set up than a tiered hierarchy'as it gives senior doctors more approachability, and helps prevent junior doctors from making poor decisions. According to one ED senior house officer, 'the culture and/or personality of the people in charge' influences the way that EDs as a whole respond to the 4-hour target, or set the risk threshold for discharging patients from the ED. The role of senior nurses was also mentioned by some participants, particularly in regard to the variety of approaches they can take 'Depending on who the charge nurse is, some are a little more relaxed, other as soon as you hit 3 hours they're on to you' (senior house officer).

According to the informants, there are differences in departmental cultures across the three sites. While informants from site 1 were mostly positive about the culture of management and work ('I think we're a very balanced fair department' (ED senior house officers) and 'the culture is very healthy and morale is high' (ED consultant)), there were more critical comments about culture on site 2 ('(culture) could be better in the department' (senior house officer) and 'the stresses of the department can cascade down, it's not always as supportive there' (manager)).

The nature of the working relationship between the ED and other hospital departments was also described as influencing admitting practices: 'Very much depends on who's on the end of the phone, some (medical registrars) will be dismissive and say that's fine you can send them home their GP can sort that out, or that doesn't sound serious to me and before you know it someone with something quite serious has been sent home. In other ways there like whatever you probably haven't done anything of course we'll come and see them. Then they're not very good at sending them home so they end up getting admitted.' (ED consultant).

\section{Responding to patient preferences and expectations}

A final issue raised by informants about the potential avoidability of admissions from the ED concerned the way in which ED doctors respond to patient expectations and preference regarding admission or discharge. It was explained that decisions to discharge were often unpopular with patients and their family and that this may eventually result in some clinicians deciding to admit the patient as a consequence. According to one ED registrar, 
'patients' expectations are getting higher and higher, and I think patients want more'. It was felt by some informants that more could be done to challenge patient preferences for being admitted when there may not be clinical grounds for doing so. One ED consultant, for example, suggested that the ED could do more to 'change the perception you've been discharged rapidly' and to emphasise that 'stays in hospital are a major risk'.

\section{DISCUSSION}

This qualitative study is, to our knowledge, the first to systematically explore consultant, junior doctor, nurse and manager perceptions and views about those factors potentially contributing to avoidable or unnecessary hospital admission from EDs. Although data were derived from a relatively small number of informants across three sites, the study has illuminated a number of factors that appear to be contributing to avoidable or unnecessary admissions and which deserve further consideration by hospital and ED managers.

One of the reasons for conducting this study was to identify any potential reasons as to why the site $2 \mathrm{ED}$ had a higher conversion rate than the other two EDs even after controlling for age, race, level of deprivation, arrival by ambulance and time of arrival. While our study was not designed to demonstrate a statistical relationship between any factors influencing admissions practice and the actual conversion rate of the three sites, it has generated some hypotheses as to why there may be a higher rate of admission in one site compared with others.

However, the most prominent issue raised by our informants was in fact not site-specific. According to our informants, the national 4-hour waiting target, which places a pressure on clinicians to quickly move patients out of the ED (several informants made reference to the ' 3 hour 59 min' patient), was an underlying cause for unnecessary or avoidable admissions. These findings correspond to national data that have shown that $24 \%$ of admissions from EDs occur in the last $10 \mathrm{~min}$ before a patient breaches the 4-hour target. ${ }^{3}$

While most informants noted that the 4-hour target had many positive effects in terms of improving hospital efficiency and reducing waiting times for patients, there was a view that some admissions could be avoided if there was not such a strict and inflexible adherence to the target. Although studies have also found that introduction of the 4-hour target has not increased the overall proportion of admissions from the ED, there are many potential confounders involved in the relationship between admission rates and the 4-hour target. ${ }^{10}$ Several key studies have shown a positive effect of the 4-hour target on patient care. A study examining compliance to the Australian National Emergency Access Target revealed reduced inpatient hospital mortality with increased compliance to the target. In addition, a study of 15 EDs in the UK over a 3-year period showed no negative effect on the quality or safety of ED care with the introduction of the 4-hour target. However, the findings from our research support earlier calls for more research on the 4-hour target, possibly involving the piloting of policy modifications that may prevent unnecessary or avoidable admissions. ${ }^{10-13}$

The full impact of the 4-hour target on admission rates and the overall quality of care has not been adequately studied, and the findings from our research support earlier call for more research on the 4-hour target, possibly involving the piloting of policy modifications that may prevent unnecessary or avoidable admissions.

When it came to factors that are more under the direct control of hospitals and EDs, the level of experience and confidence of doctors was identified as being important in avoiding unnecessary admissions. Increased senior input into admission decisions has previously been shown to have an impact on standardised admission ratios as well as on hospital standardised mortality ratios. ${ }^{14}$ Linked to this was also the mention of a culture of good communication between junior and more senior doctors. As a result, increasing access to senior clinicians, through both increased availability and accessibility in terms of rapport with junior members of staff, was felt to be one way of reducing admission rates from the $\mathrm{ED}$, and should be an area of further inquiry.

The availability of specialty expertise was not a prominent factor mentioned by our informants even though evidence from trials of providing rapid access to geriatricians, physiotherapists and occupational therapists have shown moderate success in avoiding admissions, including one trial at Newham hospital run by A\&E nurses with support from physiotherapists and occupational therapists which succeeded in avoiding a number of admissions during the study period. ${ }^{15}$

There was also no mention of a high reliance on temporary locum staff by EDs being associated with higher rates of admission, even though one might reasonably expect short-term locums who are unfamiliar with the ways an ED operates to lead to a higher rate of admission, especially when combined with the pressure of the 4-hour waiting target. We did not measure the dependency on short-term locum staff in the three sites, but this might be worth exploring in further future research.

A second set of factors under the control of hospitals and EDs which were raised by our informants relate to a number of adjunct services that, if available and accessible, could help avoid a number of admissions. Of particular note were the availability of diagnostic services and social care packages. Several informants talked about patients being admitted because they could not access diagnostic services in time to avoid a 4-hour breach, or because they did not trust the system to allow patients to be discharged from the ED and then be brought back quickly for a rapid outpatient appointment. Informants also mentioned the lack of access to a patient's past clinical notes as being an occasional hindrance to a full clinical assessment which might, in some cases, have resulted in patients being discharged home rather than admitted. 
Similarly, a lack of effective liaison with social services and the inability to arrange for home-based care was said to have resulted in a number of admissions that were potentially avoidable or unnecessary. There is some evidence that the availability of effective community-based social services can help reduce the overall incidence of emergency admissions of frail and elderly people in the community. ${ }^{16}$ However, it seems unlikely that EDs can do much more to avoid admissions by making arrangements for home care given the 4-hour waiting target.

Informants also noted that some unnecessary and avoidable admissions were the result of patients expressing a strong personal preference for admission, even when not necessary from a clinical perspective. Although these social reasons might be described as being patient-related factors, it was suggested by some informants that the frequency of admissions could be reduced if more were done to educate and persuade patients about the risks associated with unnecessary hospital admission.

Clearly, many of the factors raised by our informants do not operate in isolation. Factors that have been shown to enable a more efficient throughput of patients include a combination of early senior assessment, frontloading of investigations, training nursing staff to order X-rays, ensuring the right mix of skills and competencies, streaming of broadly similar patient groups, streamlining transfer policies, developing ambulatory care pathways to avoid admission and sending well patients home to await results that are anticipated to be normal with telephone follow-up. ${ }^{17}$

Ensuring that EDs provide the right combination of staff, services and interventions aimed at reducing avoidable and unnecessary admissions point to the importance of effective management at the level of both hospital and ED. However, studying the impact of the quality of management on hospital or ED performance is a difficult but neglected area of study. However, the data from our informants note the importance of organisational culture and effective management on clinical practice in EDs, and suggest that the site with the highest conversion rate is also the site perceived to have the highest level of frustration and demotivation among its staff.

The construction of rigorous observational or experimental studies to study the impact of improvements in 'management' or 'organisational culture' is logistically and methodologically challenging. However, the findings from this research are sufficiently strong enough to lead each of the three hospitals to review and assess their current practices with the aim of implementing interventions to improve the performance of their EDs, including reducing the number of unnecessary or avoidable admissions. In particular, it provides to a number of areas that the hospital with the particularly high conversion rate should look at, possibly in comparison with the practices at the other two sites.

\section{CONCLUSIONS}

National data have shown a significant variation in the admission rates between NHS hospitals which cannot be fully accounted for by differences in demographics and clinical need. The management, senior support, processes, pathways and culture of the ED also play a key role in determining admission behaviour and rates. In order to reduce unnecessary or avoidable admissions, it is important to assess and understand how EDs are managed and organised. This paper describes the findings of a qualitative study that was designed to rapidly identify those factors believed to be contributing to unnecessary or avoidable admissions.

The precise contribution of these different factors could be evaluated quantitatively by other studies. Multiple areas of future study have been identified including: the role of the 4-hour target in admission behaviour and how this can be mitigated, the role of ED staffing and particularly access to senior support, the incidence and possible preventability of 'social admissions', and the importance of the relationship between primary and secondary care in avoiding admission. But in addition to this, the findings from this study can prompt local management and policy decisions aimed at improving performance which can then be accompanied by inhouse monitoring and evaluation to enable further ongoing review and reassessment.

Contributors IP, DM and TH came up with initial idea for the study. IP and DM developed the questionnaires. IP recruited and carried out the interviews. IP, HB, DM and SAI transcribed and analysed the interviews. IP, HB and SAl drafted and revised the manuscript with input from DM and TH.

Competing interests SAI, IP, DM and TH have support from Barts Health NHS Trust for the submitted work. Other authors have no competing interest to declare.

Ethics approval Queen Mary University of London Research Ethics Committee and the Barts Health Research and Development Committee.

Provenance and peer review Not commissioned; externally peer reviewed.

Data sharing statement Extra data can be accessed via the Dryad data repository at the doi:10.5061/dryad.5m2c3.

Open Access This is an Open Access article distributed in accordance with the Creative Commons Attribution Non Commercial (CC BY-NC 4.0) license, which permits others to distribute, remix, adapt, build upon this work non-commercially, and license their derivative works on different terms, provided the original work is properly cited and the use is non-commercial. See: http://creativecommons.org/ licenses/by-nc/4.0/

(c) Article author(s) (or their employer(s) unless otherwise stated in the text of the article) 2017. All rights reserved. No commercial use is permitted unless otherwise expressly granted.

\section{REFERENCES}

1. Royal College of Physicians. Hospitals on the edge? The time for action, 2012.

2. Blunt I. Focus on preventable admissions. London: The Health Foundation/Nuffield Trust, 2013.

3. National audit office. Emergency admissions to hospital: managing the demand, 2013.

4. O'Cathain A, Knowles E, Maheswaran R, et al. A system-wide approach to explaining variation in potentially avoidable emergency admissions: national ecological study. BMJ Qual Saf 2014;23:1-9.

5. O'Cathain A, Knowles E, Turner J, et al. Variation in avoidable emergency admissions: multiple case studies of eme 
rgency and urgent care systems. $J$ Health Serv Res Policy 2016;21:1-10.

6. Calder LA, Forster AJ, Stiell IG, et al. Mapping out the emergency department disposition decision for high-acuity patients. Ann Emerg Med 2012;60:567-76.

7. Purdy S. Avoiding hospital admissions what the evidence says. The King's Fund, 2012.

8. Department of Health reforming emergency care. London: HMSO, 2001.

9. Mortimore A, Cooper S. The "4-hour target": emergency nurses' views. Emerg Med J 2007;24:402-4.

10 Boyle A, Mason S. What has the 4-hour access standard achieved? Br J Hosp Med 2014;75:620-2.

11 Weber EJ, Mason S, Freeman JV, et al. Implications of England's four-hour target for quality of care and resource use in the emergency department. Ann Emerg Med 2012;60:699-706.
12 Sullivan C, Staib A, Khanna S, et al. The National Emergency access target (NEAT) and the 4-hour rule: time to review the target. Med $J$ Aust 20162016;204:354;204:354.

13. Jones $\mathrm{P}$, Chalmers L, Wells $\mathrm{S}$, et al. Implementing performance improvement in New Zealand emergency departments: the six hour time target policy national research project protocol. BMC Health Serv Res 2012;12:45.

14. Boyle A, Beniuk K, Higginson I, et al. Emergency department crowding: time for interventions and policy evaluations. Emerg Med Int 2012;2012:1-8.

15. Crane K, Sparks L. An admission avoidance team: its role in the Accident \& Emergency department. Accid Emerg Nurs 19991999;7:91-5;7:91-5.

16. NHS England. What actions can be taken to reduce emergency admissions?

17. Boyle AA, Ahmed V, Palmer CR, et al. Reductions in hospital admissions and mortality rates observed after integrating emergency care: a natural experiment. BMJ Open 2012;2:e000930. 